\title{
PLUGGING FLOWS
}

BY

PETER B. PERCELL AND F. WESLEY WILSON, JR.

\begin{abstract}
A plug construction is a local modification of a nonsingular flow which severs certain kinds of recurrence properties. In this paper we investigate the effect of plug constructions on minimal sets, the nonwandering set, and the chain recurrent set and the explosions of these sets when a plug construction is perturbed.
\end{abstract}

1. Let $\xi$ be a nonsingular $C^{r}$ vector field on a smooth manifold $M^{n}(n>3)$. In previous papers [1] and [2], we have used local (plug) modifications of $\xi$ to obtain a new vector field $\zeta$ which is homotopic to $\xi$ and which has much more simple recurrence properties. However in [3], we discover that under perturbations of $\zeta$, the recurrence properties of $\xi$ may reappear. In this paper, we describe some new examples based on plug constructions, and we gain some insight into the relationship between plug constructions and recurrence. In particular, we obtain some examples which lead to interesting comparisons of the nonwandering set and the chain recurrent set. We have an example where the chain recurrent set explodes, but the nonwandering set does not. Thanks are due to Charles Conley for pointing out the usefulness of chain recurrence in studying these examples.

We shall use the following notational conventions in this paper. Let $\xi$ be a nonsingular vector field on $M$. By a homotopy of $\xi$ we shall always mean a homotopy through nonsingular vector fields. Since we shall be dealing with compact manifolds and $C^{r}$ vector fields $(1<r<\infty)$, it follows that $\xi$ induces a $C^{r}$ action of the additive reals on the manifold. This action is customarily called the flow generated by $\xi$. We shall abuse notation by also letting $\xi: M \times \mathbf{R} \rightarrow M$ denote the flow generated by the vector field $\xi$.

1. The plug construction. A flow box for $\xi$ is a coordinate neighborhood $U$ in $M$ with coordinates $\left(x_{1}, x_{2}, \ldots, x_{n}\right)$ such that $a<x_{1} \leqslant b$ and $x_{1}$ is the time parameterization of the flow which is induced by $\xi$, i.e. $\xi \mid U=\partial / \partial x_{1}$. Usually $U$ is a topological $n$-ball. The ingress region of $U$ is designated by $U^{+}$and the egress region is designated by $U^{-}$. These sets are diffeomorphic by the flow. $\xi$ is tangent to $U$ along the remainder of its boundary $\left(\partial U-U^{+}\right.$

Received by the editors February 12, 1976.

AMS (MOS) subject classifications (1970). Primary 34C99, 58F99.

Key words and phrases. Periodic solution, nonwandering set, chain recurrent set.

- American Mathematical Society 1977 
$\left.\cup U^{-}\right)$, and we call this set the sides of $U$.

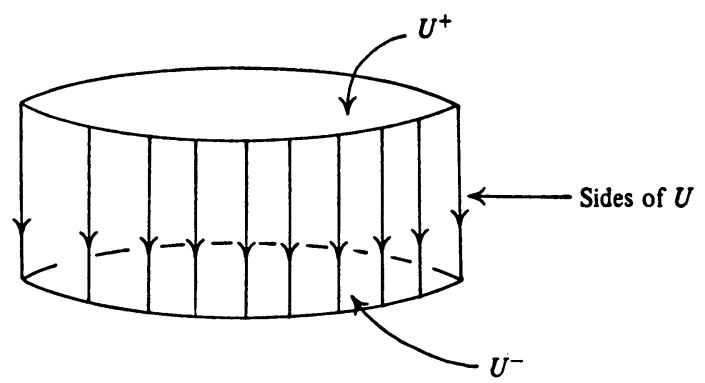

Figure 1. A flow box

Let $V \subset U$ be a closed set which is the union of full trajectory segments, and such that $V^{+}=V \cap U^{+}$is a subset of the interior of $U^{+}$.

Definition 1.1. A plug for $(U, V)$ is a nonsingular vector field $\zeta$ on $U$ such that

$1 . \zeta$ coincides with $\xi$ near $\partial U$.

2. Every $\zeta$ trajectory which begins in $V^{+}=V \cap U^{+}$(respectively, in $V^{-}=V \cap U^{-}$) remains in $U$ for all positive time (negative time, respectively).

3. If $\gamma$ is a $\zeta$ trajectory which leaves $U$ in positive and in negative time, then $\gamma \cap U$ coincides with one of the $\xi \mid U$ fibers in some neighborhood of $\partial U$.

In [1], it is shown that there are smooth plugs which have four minimal sets which are $k$-dimensional tori $(1<k<n-2)$ on which the restricted flow is dense, and which have a hyperbolic structure in the complementary dimensions. These are the only limit sets of $\zeta \mid U$. The relationship of the flow and these minimal sets is analogous to the relationship of the flow and the singular points in Figure 2(a). We note that by coalescing the upper pair and the lower pair, we can obtain an example which no longer has a hyperbolic structure and which has only two minimal sets (Figure 2(b)). We shall usually be thinking of this simple plug, throughout this paper.

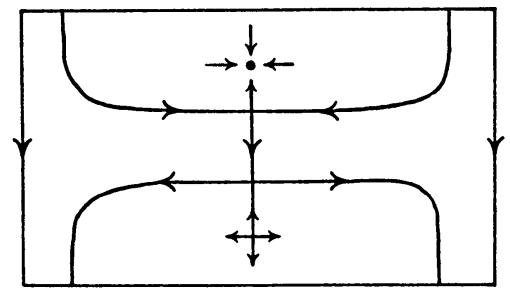

(a) Almost Morse-Smale plug

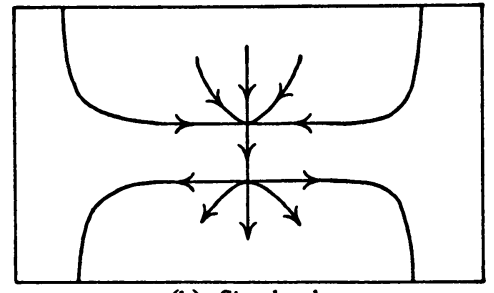

(b) Simple plug

FIGURE 2. Some 2-dimensional plugs

The third property of a plug is satisfied if the plug satisfies the mirror-image 
property. If $-a \leqslant x_{1} \leqslant a$, then we can express this property in coordinates, cf. [1]:

$$
\begin{aligned}
& \zeta_{1}\left(-x_{1}, x_{2}, \ldots, x_{n}\right)=\zeta_{1}\left(x_{1}, x_{2}, \ldots, x_{n}\right), \\
& \zeta_{k}\left(-x_{1}, x_{2}, \ldots, x_{n}\right)=-\zeta_{k}\left(x_{1}, x_{2}, \ldots, x_{n}\right) \text { for } k=2, \ldots, n .
\end{aligned}
$$

In addition to [1] and [2], there are two other examples of uses of plugs in the literature. The first of these is the example given by Brock Fuller [4] of a flow on a solid torus which is transverse at the boundary and for which the only periodic solution is contractible. Fuller did not view this as a plug construction, but we shall now describe it in this way. Let us begin with the flow on the solid torus which is transverse (inwards) at the boundary and which has one periodic solution which is asymptotically stable and which attracts the whole solid torus. Let $U$ be a flow box which is centered on the periodic solution, and let $V$ denote the contraction of $U$ which results from following the flow forward once around the solid torus. We can build a Fuller-type example which has two periodic solutions by plugging $(U, V)$. However, in this case the contracton of the flow is so strong that the third property of the plug is not used. We can obtain the original Fuller example (with just one periodic solution) by using a weaker version of the plug for $(U, V)$ which only satisfies properties 1 and 2 .

The other example is P. Schweitzer's construction [5] of a $C^{1} 3$-dimensional plug which is not a 3-ball and which does not contain any periodic solutions, only Denjoy minimal sets. This construction yields a plug which does not absorb an open set $(V)$. However, by [1], any nonsingular flow is homotopic to a flow which has only finitely many periodic solutions, and so by inserting Schweitzer's plugs to sever these, one obtains examples of $C^{1}$ flows on every 3-manifold.(and in every homotopy class) which have only Denjoy minimal sets.

In general, a plug will absorb (positively) a subset $A^{+}$of $U^{+}$and (negatively) a subset $A^{-}$of $U^{-}$. It is always the case that $A^{+}$and $A^{-}$are diffeomorphic by the parallel flow on $U$. In Schweitzer's construction, $A^{+}$has no interior. In each of the other examples $A^{+}$is an $(n-1)$-manifold with boundary. We shall call the global saturation of $\partial A^{+}$the attracted manifold of $U$, and the global saturation of $\partial A^{-}$. the repelled manifold of $U$. For the almost Morse-Smale plugs, these sets are indeed the attracted and repelled manifolds of saddles in $U$. For the simple plug, they play a similar role. We shall also have occasion to refer to the respective saturations of $A^{+}$and of $A^{-}$. We shall denote these sets respectively $U^{a}(\zeta)$ and $U^{r}(\zeta)$ and call them the attracted region of the plug $P=(U, \zeta)$ and the repelled region of $P$.

We conclude this section with a discussion of the homotopy properties of plug constructions. Clearly $\zeta \mid U$ is not even $C^{0}$ close to $\zeta \mid U$. However, they 
are homotopic rel( $\partial U)$. To see this, we observe that the simple plug construction has no vertical upwards vector, and so its convex combination with $\xi \mid U$ $(s \zeta+(1-s) \xi)$ gives a nonsingular homotopy. On the other hand, an almost Morse-Smale plug (Figure 2(a)) is clearly homotopic to a simple plug (Figure 2(b)).

2. Plug-saturation. The other idea in [1] is that if $M$ is compact, then there is a finite family of flow boxes $\left\{U_{i}\right\}_{i=1}^{k}$ for $\xi$ with corresponding subflow boxes $V_{i} \subset U_{i}$ such that every $\xi$ trajectory intersects the interior of some $V_{i}$ in positive time and of some $V_{j}$ in negative time [1, Theorem A]. Let $\zeta$ be a vector field on $M$ such that $\zeta\left|M-\cup_{i=1}^{k} U_{i}=\xi\right| M-\cup_{i=1}^{k} U_{i}$ and such that $\zeta_{i}=\zeta \mid U_{i}$ is a plug construction for $\left(U_{i}, V_{i}\right)$. Then $\left\{P_{i}=\left(U_{i}, \zeta_{i}\right)\right\}$ is called a plug-saturation for $\xi$. $\zeta$ has the property that every trajectory enters the interior of some $A_{i}^{+}$(of some $A_{i}^{-}$) in finite positive (negative, respectively) time. Thus a plug-saturation $\zeta$ of $\xi$ has the property that the only $\alpha$ - and $\omega$-limit sets of $\zeta$ are the minimal sets in the plugs [1].

LEMMA 2.1. If $\zeta$ is a plug-saturation of $\xi$ with plugs $\left\{P_{i}=\left(U_{i}, \zeta_{i}\right)\right\}_{i=1}^{k}$, then there is a positive bound $T$ such that every $\xi$ trajectory originating in $M-$ $\bigcup_{i=1}^{k} U_{i}$ intersects the interior of some $A_{i}^{+}$in positive time $<T$ and intersects the interior of some $A_{i}^{-}$in negative time $>-T$.

Proof. Suppose that there is a convergent sequence of points $\left\{x_{n}\right\}_{n=1}^{\infty}$ and a properly divergent sequence of positive times $\left\{t_{n}\right\}_{n=1}^{\infty}$ such that the trajectory segment from $x_{n}$ with positive length $t_{n}$ fails to intersect any $A_{i}^{+}$. Let $x_{0}=\lim x_{n}$. Now the positive semitrajectory from $x_{0}$ enters the interior of $A_{i}^{+}$ in time $t_{0}$. By continuity, the positive semitrajectories from $x_{n}$ enter the interior of ${A_{i}}^{+}$in times less than $t_{0}+1$, contrary to the choice of $\left\{\left(x_{n}, t_{n}\right)\right\}$. Thus a positive bound $T$ must exist. Similarly, a negative bound must exist.

We call a plug-saturation for $\xi$ an $\Omega$-saturation if $\zeta$ has the property that its nonwandering set $\Omega$ is the union of the finite collection of minimal sets in the plugs. In particular, a plug-saturation is an $\Omega$-saturation if it has the property that no positive trajectory segment of $\zeta$ joins $U_{i}^{-}$to $U_{i}^{+}$. Using this observation and looking carefully at the proof of [1, Lemma 2.4], we see that the following result is actually proved in [1].

THEOREM 2.2. Let $\xi$ be a nonsingular $C^{r}$ vector field on the compact manifold $M^{n}(1<r<\infty)$. Then there is a plug construction $\zeta$ for $\xi$ with respect to plugs $\left\{P_{i}=\left(U_{i}, \zeta_{i}\right)\right\}_{i=1}^{k}$ such that

1. $\zeta \simeq \xi \operatorname{rel}\left(M-\cup_{i=1}^{k} U_{i}\right)$;

$2 . \zeta$ is an $\Omega$-saturation.

Defintion 2.3 (cf. [3]). $\zeta$ is an almost Morse-Smale vector field if $\zeta$ has a 
finite number of periodic solutions, they are all hyperbolic, and their union is $\Omega$.

COROLlaRY 2.4. If $\xi$ is a nonsingular $C^{r}$ vector field on a compact manifold $M(1<r<\infty)$, then $\xi$ is homotopic to an almost Morse-Smale vector field.

Proof. Apply the theorem using almost Morse-Smale plug constructions.

THEOREM 2.5 (cf. $[6,88])$. Let $\xi$ be a $C^{r}$ vector field on a compact manifold $M^{n}(1<r<\infty)$. Then there is a plug construction $\zeta$ with respect to the single plug $P=(U, \zeta \mid U)$ such that

$1 . \zeta \simeq \xi \operatorname{rel}(M-U)$,

2. $P$ is the analogue of one of the examples in Figure 2,

3. $\{P\}$ is a plug-saturation of $\xi$,

4. $\Omega_{\zeta}$ is the invariant cylinder of $\zeta \mid U$ which is bounded by the two periodic solutions in the case of the simple plug, or in the case of an almost MorseSmale plug, $\Omega_{\zeta}$ is the union of the four periodic solutions and of the invariant cylinder which is bounded by the saddles.

Proof. By [1, Theorem A], we can find a finite plug-saturation $\zeta_{0}$ with plugs $\left\{P_{i}=\left(U_{i}, \zeta_{i}\right)\right\}_{i=1}^{k}$. Let $\gamma$ be an arc in the complement of the plugs which joins the side of $U_{1}$ to the side of $U_{2}$. By transversality theory [7], $\gamma$ can be chosen so that $T \gamma$ is transverse to $\xi$ in $T M$. Since $n>3$, this means that $\xi$ is never tangent to $\gamma$. Thus we can find an embedded ribbon $\gamma \times I$ in the complement of the plugs so that $\gamma \times 0$ is an arc in the side of $U_{1}$, so that $\gamma \times 1$ is an arc in the side of $U_{2}$, and so that $\gamma \times I$ is transverse to $\xi$. This ribbon can be thickened by trajectory segments so that $U_{1} \cup U_{2} \cup(\gamma \times I \times$ $I$ ) is a flow box, cf. Figure 3.

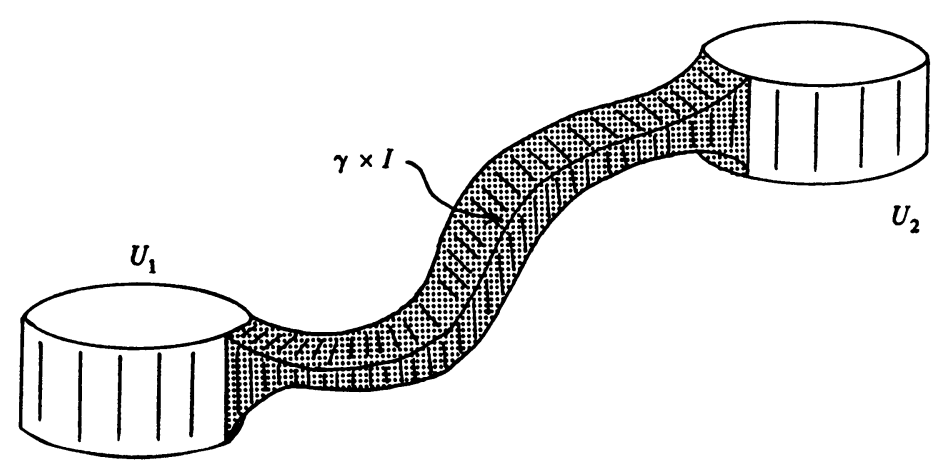

FIGURE 3. Joining two flow boxes

Continuing inductively, we can construct a single flow box $U$ which contains $\cup_{i=1}^{k} U_{i}$. Note that the ingress and egress regions of $U$ contain the 
respective ingress and egress regions of each $U_{i}$, and that there is a plug construction $\zeta \mid U$ so that the regions $A^{+}$and $A^{-}$include the regions $\cup_{i=1}^{k} A_{i}^{+}$ and $\cup_{i=1}^{k} A_{i}^{-}$, respectively. This means that $(U, \zeta)$ is a plug-saturation of $\xi$ and also that every point exterior to $U$ is wandering. Thus $\Omega_{\zeta}$ is a closed invariant set which is a subset of $U$. The following lemmas are useful for the determination of $\Omega_{\zeta}$.

LEMMA 2.6. The attracted and repelled manifolds of $P$ have nonempty intersection.

PROof. If they have empty intersection, then the forward image of $A^{-}$is a proper subset of the interior of $A^{+}$, and so $U^{r}(\zeta) \cup U$ is a closed region in $M$. This region is not all of $M$, since it does not contain points which are carried into $\partial A^{+}$in positive time. Thus $P$ fails to be a plug-saturation of $\xi$.

LEMMA 2.7. $P=(U, \zeta)$ can be chosen so that the attracted and repelled manifolds of $P$ intersect transversely.

Proof. By Lemma 2.1, there is a time $T$ such that each point in $\partial A^{-}$is carried by $\xi$ into the interior of $A^{+}$in time $<T$. In particular, there is no trajectory which originates in $\partial A^{-}$and which intersects $\partial A^{+}$directly above $\partial A^{-}$without first intersecting the interior of $A^{+}$, i.e. for each point $p \in \partial A^{-}$ which is mapped into $\partial A^{+}$by the positive $\zeta$-flow (say in time $t_{0}<T$ ), there is a $\partial A^{-}$neighborhood $W_{p}$ of $p$ such that the map of $W_{p}$ into $U^{+}$in time approximately $t_{0}$ has its image nowhere above $W_{p}$. On the other hand, if $p \in \partial A^{-}$is carried into $U^{+}$in time $t_{1}<T$, but the image point is not in $\partial A^{+}$, then there is a neighborhood $W_{p}$ of $p$ in $\partial A^{-}$such that the map of $W_{p}$ into $U^{+}$in time approximately $t_{1}$ has its image nowhere above $W_{p}$. Consider the closed subset of $\partial A^{-} \times[0, T]$ consisting of those pairs $(p, t)$ such that the $\zeta$ flow translation of $p$ for time $t$ lies in $U^{+}$, but such that no such translation for a shorter time lies in $A^{+}$. This set is covered by open sets of the form $W_{p} \times(t-\sigma, t+\varepsilon)$ where $\varepsilon$ is smaller than the time length of $U$, and hence has a finite subcover $\left\{W_{i} \times\left(t_{i}-\varepsilon, t_{i}+\varepsilon\right)\right\}_{i=1}^{k}$. Let $V_{i} \subseteq W_{i}$ be chosen so that $\bar{V}_{i} \subseteq W_{i}$ and so that $\left\{V_{i} \times\left(t_{i}-\varepsilon, t_{i}+\varepsilon\right)\right\}_{i=1}^{k}$ is still a covering. By a standard relative transversality argument, we can find a small isotopic perturbation of $\partial A^{-}$so that the isotopy is the identity on the complement of $W_{i}$ and so that the map of $V_{i}$ into $U^{+}$in approximately time $t_{i}$ is transversal to $\partial A^{+}$. Using the flow isotopy construction of [8, Theorem 5.6] and of [9] we can alter $\zeta$ in $U$ so that $(U, \zeta)$ is still a plug, but so that $\partial A^{-}$has been altered by this isotopy. Note that $\partial A^{+}$has also been altered, but not near the target area for $W_{i} \times\left(t_{i}-\varepsilon, t_{i}+\varepsilon\right)$. Using the openness of transversality we can repeat this process inductively to obtain the result of this lemma.

We continue the proof of the theorem by assuming that $\zeta$ has been chosen 
so that the attracted and repelled manifolds of $\zeta$ have transversal intersection. If $P$ is an almost Morse-Smale plug, then we have a cycle of intersecting invariant manifolds of hyperbolic periodic solutions, and every intersection except for the one in the plug is transverse. Thus by the Cloud Lemma [10], it follows that $\Omega_{\zeta}$ is the union of the periodic solutions in the plug, together with the invariant cylinder which is the pair of coincident invariant manifolds. To see that $\Omega_{\zeta}$ has the analogous form for the choice of a simple plug, we observe that during the homotopy between Figure 2(a) and Figure 2(b), the attracted and repelled regions are affected only regarding their internal structure, and consequently the only change in $\Omega$ during the homotopy is the obvious coalescence of the periodic solutions.

REMARK. Lemma 2.7 could have been proved as an application of Percell's Condition G [11]. However, since the present context is slightly different from his previous context, the required adaptations are more complicated than the proof which we have given. Essentially what we have done is to reestablish the density of condition $G$ in this rather simple case.

3. Some consequences of the one-plug construction. The examples obtained in Theorem 2.5 have some interesting consequences. By choosing simple plugs, we have

THEOREM 3.1. Let $M$ be a compact $n$-manifold with Euler characteristic zero $(n \geq 3)$. Then every nonsingular vector field on $M$ is homotopic to a nonsingular vector field which has just two periodic solutions. They are unknotted, unlinked, and have index zero. Moreover they are the $\alpha$-and $\omega$-limit of every other trajectory in $M$. Finally, they are the common boundary of an invariant cylinder which is the full nonwandering set of this vector field.

This theorem shows that the usual algebraic invariants of periodic solutions cannot be used to determine the homotopy class of a vector field. The analogous result with an almost Morse-Smale plug has four hyperbolic periodic solutions.

Recently, several people have been studying the "Poincaré mapping" between local cross sections of a flow to obtain information about recurrence [12], [13], and [14]. In the one-plug examples, the sets $U^{-}$and $U^{+}$are cross sections which intersect every trajectory. Moreover, if we totally understood the Poincare mapping between these cross sections, then we would have full information about the flow. This is really a negative result, for it says that the problem of classifying all Poincaré mappings is as difficult as the problem of classifying all nonsingular flows.

Another interpretation of the one-plug examples can be given in terms of separatrix behavior [15] and [16]. If $\zeta$ is a one-plug example with simple plug $P=(U, \zeta)$, then the flow induced by $\zeta$ is elliptic with respect to the cylinder 
$\Omega_{\xi}$, i.e. every trajectory has its $\alpha$ - and $\omega$-limit at $\Omega_{\xi}$. If $p$ is a point in $M-\Omega_{\xi}$ which does not lie on either the attracted or repelled manifolds of $P$, then there is a neighborhood of $p$ all of whose trajectories go from $A^{-}$to $A^{+}$in about the same amount of time, i.e. the separatrices of $\zeta$ are the union of the attracted and repelled manifolds of $P$. Under the hypothesis that these manifolds intersect transversely, this separatrix set satisfies a natural "manifold hypothesis", cf. [15]. It seems possible that this viewpoint may be useful for unravelling the mysteries of the manifold hypothesis.

4. Chain recurrence. By this time, one begins to be impressed that the plug construction should not be allowed to work. It is rather unreasonable that a local homotopy-modification of a flow can alter the qualitative properties so violently. But in fact it does work, which we interpret as an indictment of the measures of qualitative behavior which we habitually use. We shall conclude this paper by discussing a notion of recurrence which was recently introduced by C. Conley [17], and which has the interesting property that it remains unaffected by simple plug constructions.

Definition 4.1. An $\varepsilon$-chain for $\xi$ is a collection

$$
\left\{x_{1}, \ldots, x_{n+1}, t_{1}, \ldots, t_{n} \mid x_{i} \in M, t_{i}>1\right\}
$$

and such that the distance from $\xi\left(x_{i}, t_{i}\right)$ to $x_{i+1}$ is less than $\varepsilon$ for $i=1, \ldots, n$. The point $x$ is called chain recurrent if for every $\varepsilon>0$, there is an $\varepsilon$-chain such that $x_{1}=x_{n+1}=x$.

We shall denote the chain recurrent set of $\xi$ by $\Gamma_{\xi}$. It is clear that $\Gamma_{\xi}$ is a closed invariant set and that $\Omega_{\xi} \subset \Gamma_{\xi}$, cf. [17].

THEOREM 4.1. If $\zeta$ is a simple plug modification of $\xi$, then

$$
\Gamma_{\xi} \cap\left(M-\bigcup_{i=1}^{k} U_{i}\right) \subset \Gamma_{\zeta} \cap\left(M-\bigcup_{i=1}^{k} U_{i}\right) .
$$

Proof. Let $x \in \Gamma_{\xi} \cap\left(M-\cup_{i=1}^{k} U_{i}\right)$ and let $\varepsilon>0$ be given. Then there is an $\varepsilon$-chain $\left\{x=x_{1}, \ldots, x_{n+1}=\chi, t_{1}, \ldots, t_{n}\right\}$ for $\chi$ and $\xi$. We construct an $\varepsilon$-chain for $\chi$ and $\zeta$ as follows. Take $y_{1}=\chi$. If the segment $S=\xi\left(y_{1},\left[0, t_{1}\right]\right)$ intersects no plugs, then we take $t_{1}$ as before and $y_{2}=x_{2}$. Suppose $S$ intersects plugs $P_{1}, \ldots, P_{k}$ (in this order). There are two cases: (I) $S$ enters $P_{1}$ and is perturbed on the interior of $P_{1}$, but exits from $P_{1}$ to continue on its way, or (II) a portion of $S$ is attracted in $P_{1}$, cf. Figure 4.

In (I) there is a time difference $\tau_{1}$ between the time that it takes to get from $S \cap U_{1}^{+}$to $S \cap U_{1}^{-}$along $\xi$ and along $\zeta$. In this case, we choose $y_{1}=\chi$ but we augment $t_{1}$ by $\tau_{1}$ and continue on $P_{2}$. In case (II), we can 


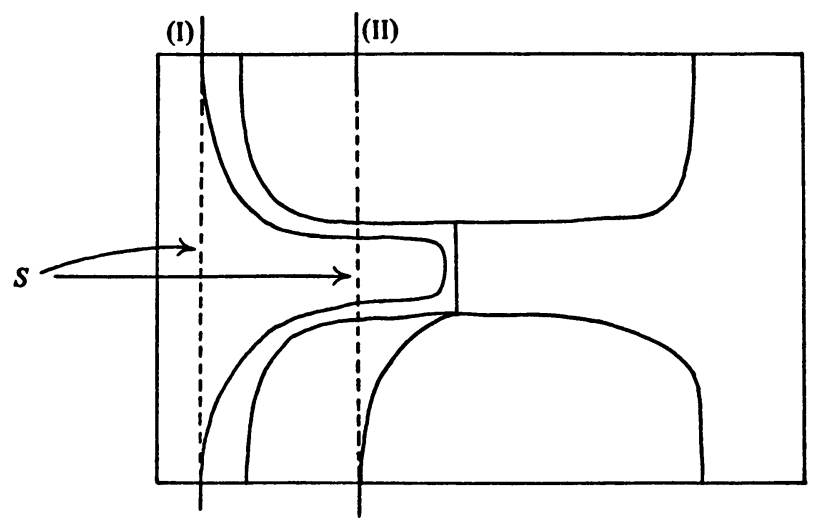

FIGURE 4. A plug intersecting a trajectory segment

choose $t_{1}^{\prime}$ so large that $\zeta\left(y_{1}, t_{1}^{\prime}\right)$ lies within $\varepsilon / 2$ of the attracting periodic solution in $P_{1}$. Choose $y_{2}$ to lie on the invariant cylinder in $P_{1}$, and within $\varepsilon$ of $\zeta\left(y_{1}, t_{1}^{\prime}\right)$. Choose $t_{2}^{\prime}>1$ so that $\zeta\left(y_{2}, t_{2}^{\prime}\right)$ lies within $\varepsilon / 2$ of the repelling periodic solution in $P_{1}$, and choose $y_{3}$ to lie on the trajectory which again becomes coincident with $S$ and to lie within $\varepsilon$ of $\zeta\left(y_{2}, t_{2}^{\prime}\right)$, cf. Figure 5. The choice of $t_{3}^{\prime}$ depends on what happens at $P_{2}$, etc., but it is clear how we can repeat this process to construct the desired $\varepsilon$-chain to $\chi_{2}$, and on back to $\chi_{\text {. }}$

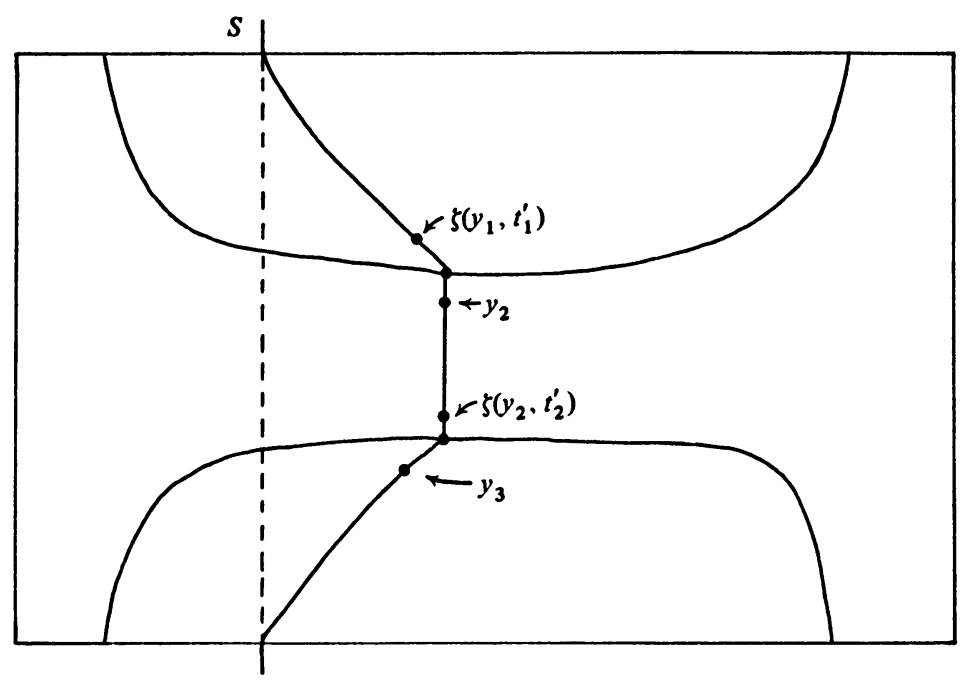

FIGURE 5. Continuing an $\epsilon$-chain through a plug

Note that this argument does not work for an almost Morse-Smale plug, since $\varepsilon$-chains cannot escape from the regions of attraction of the asymptotically stable periodic solutions. 
Let $\left\{\zeta_{\alpha}\right\}_{-1<\alpha<1}$ be a one-parameter family of vector fields. An $\Omega$-explosion ( $\Gamma$-explosion) occurs at $\alpha=0$ if there is a discontinuous increase in $\Omega(\Gamma$, respectively) as the parameter $\alpha$ is varied from $\alpha<0$ to $\alpha>0$. There are several studies of $\Omega$-explosions, cf. [10]. In [3], a case is examined where perturbations of plug constructions cause $\Omega$-explosions. This is not too surprising, since one expects nice perturbations of the vector field (e.g. Kupka-Smale) to lead to $\Gamma=\Omega$, and since $\Gamma$ often has subsets which are transverse to the plugs, we would expect that $\Omega$ would have to grow in order to achieve equality. There is a slight hole in this argument, due to the fact that the almost Morse-Smale plugs can destroy part of $\Gamma$ in certain circumstances. For example, in [3] there is an example of a plug construction such that every Kupka-Smale perturbation is Morse-Smale. For this plug construction, it is the case that $\Gamma_{\xi}$ has been altered on the complement of the plugs.

For the present, we only wish to consider the case where an almost Morse-Smale plug construction does destroy part of $\Gamma_{\xi}$. By Theorem 4.1, the analogous construction with simple plugs would not have destroyed this portion of $\Gamma_{\xi}$ (cf. the above cited example in [3]). Let $\left\{\zeta_{\alpha}\right\}$ be the family of vector fields formed by taking $\zeta_{\alpha}$ to be a simple plug construction for $\alpha>0, \zeta_{\alpha}$ to be an almost Morse-Smale plug construction for $\alpha<0$, and the variation with $\alpha$ is precisely the homotopy between Figure 2(a) and Figure 2 (b) as $\alpha \rightarrow 0$ through negative values. Then there is no $\Omega$-explosion as $\alpha \rightarrow 0$, since $\Omega$ is controlled by the plugs. On the other hand, the almost Morse-Smale plug construction may control $\Gamma$ for $\alpha<0$ (e.g. [3]), but must fail to control $\Gamma$ at $\alpha=0$, i.e. there is a $\Gamma$-explosion in the direction transverse to the plugs. Thus it is not necessary that $\Gamma$ and $\Omega$ explode simultaneously.

\section{REFERENCES}

1. F. W. Wilson, Jr., On the minimal sets of non-singular vector fields, Ann. of Math. (2) 84 (1966), 529-536. MR 34 \#2028.

2. Singularities and periodic solutions which do not attract, J. Differential Equations 8 (1970), 488-493. MR 43 \#2300.

3. C. Pugh, R. Walker and F. W. Wilson, On Morse-Smale approximations-a counterexample, J. Differential Equations 23 (1977), 173-182.

4. F.B. Fuller, Note on trajectories in a solid torus, Ann. of Math. (2) 56 (1952), 438-439. MR 14, 556.

5. Paul A. Schweitzer, Counterexamples to the Seifert conjecture and opening closed leaves of foliations, Ann. of Math. (2) 100 (1974), 386-400. MR 50 \#8557.

6. P. Percell, Presentations of 3-manifolds arising from vector fields, Trans. Amer. Math. Soc. 221 (1976), 361-377.

7. R. Abraham and J. Robbin, Transversal mappings and flows, Benjamin, New York, 1967. MR 39 \#2181.

8. J. Milnor, Lecture on the h-cobordism theorem, Math. Notes, Princeton Univ. Press, Princeton, N. J., 1965. MR 32 \#8352.

9. M. Peixoto, On an approximation theorem of Kupka and Smale, J. Differential Equations 3 (1967), 214-227. MR 35 \#499. 
10. C. Pugh and M. Shub, The S-stability theorem for flows, Invent. Math. 11 (1970), 150-158. MR 44 \#4782.

11. P. Percell, Structural stability on manifolds with boundary, Topology 12 (1973), 123-144. MR 48 \# 1267.

12. D. Rod, Pathology of invariant sets in the monkey saddle, J. Differential Equations 14 (1973), 129-170. MR 48 \#6562.

13. R. Easton, Isolating blocks and symbolic dynamics, J. Differential Equations 17 (1975), 96-118.

14. J. Yorke, Lecture at Univ. of California, Berkeley, 1975.

15. R. Elderkin, Separatrix structure for elliptic flows, Amer. J. Math. 97 (1975), 221-247.

16. - Separatrix structure for regions attracted to solitary periodic solutions, Proc. Internat. Sympos. Differential Equations (Brown Univ., 1974) (to appear).

17. C. Conley, The gradient structure of a flow (to appear).

Department of Mathematics, University of Chicago, Chicago, Illinois 60637

Department of Mathematics, University of Colorado, Boulder, Colorado 80302 (Current address of F. W. Wilson, Jr.)

Current address (P. B. Percell): Department of Mathematics, University of Houston, Houston, Texas 77004 